\title{
3D instability of liquid films and rivulets
}

\author{
Sergey Alekseenko $0^{1,2}$, Sergey Aktershev ${ }^{1,2}$, Aleksey Bobylev ${ }^{1,2}$, Vladimir Guzanov ${ }^{1,2}$ Sergey \\ Kharlamov $^{1,2}$ and Dmitriy Markovich ${ }^{1,2}$ \\ ${ }^{1}$ Kutateladze Institute of Thermophysics SB RAS, Lavrentiev ave., 1, Novosibirsk, 630090, Russia \\ ${ }^{2}$ Novosibirsk State University, Pirogov str., 2, Novosibirsk, 630090, Russia
}

\begin{abstract}
This article presents the results of experimental and theoretical study of 3D instability of waves in liquid films and rivulets, falling down a vertical plate. The method of laser induced fluorescence was used to obtain instant shapes of three dimensional waves in a liquid films and rivulets and to investigate the regularities of formation of 3D wave patterns. In particular, during 2D-3D wave transition in the film flow, we observed a strong transverse redistribution of liquid leading to the formation of rivulets on the surface of isothermal liquid film, which is a phenomenon not described previously. The results of simulation of $2 \mathrm{D}$ and $3 \mathrm{D}$ regular waves in the single rivulet are compared with the authors' previous experimental data.
\end{abstract}

\section{Introduction}

Gravity-driven film flow of a viscous liquid down a flat plane is a well known example of convectively unstable free surface flow with instabilities of different kinds leading to the appearance and interaction of surface waves with a great variety of characteristics. Specific type of film flow whereby the fluid flows in the form of many streamlets is typically called rivulet flow, whereas an individual streamlet, bounded by two contact lines, is called a rivulet. Special attention has been paid to rivulet flows because of their practical value when used in a variety of devices in power engineering and chemical technology.

This study presents the results of experimental and theoretical investigations of instabilities in the liquid film and rivulet flows. In the first part of study, the experimental results for transition from twodimensional (2D) to three-dimensional (3D) waves in falling liquid films are presented. The method of laser induced fluorescence was used to obtain instant shapes of three dimensional waves and to investigate the regularities of formation of 3D wave patterns arising due to transverse instability of 2D waves. The regular 2D waves with prescribed frequency $f$ were forced by harmonic modulation of liquid flow rate. Distilled water, water-glycerin solution (WGS) and water-ethanol solution (WES) were used as working fluids. The obtained results were compared to the results from the published literature on the modeling of 3D wave regimes of film flow.

The second part of the study is devoted to numerical simulation of $3 \mathrm{D}$ waves on the surface of a rivulet flowing down a vertical plate. The model [1] is used for numerical simulation of the forced waves in the rivulet. The results of the simulations are compared with the authors' previous experimental data [2]. The calculations are performed only for the conditions implemented in the authors' previous experiments. The comparison shows that the applied model adequately describes the 
shape of the wave surface of a rivulet, although the wave propagation velocity and wavelength are underestimated.

\section{Experimental setup and measurement technique}

The sketch of experimental setup is shown in Fig. 1. Experiments were carried out on the vertical glass plate with dimensions $20 \mathrm{~cm}$ in transversal and $25 \mathrm{~cm}$ in vertical directions. Liquid film was formed by a slot distributor with adjustable slot width. The method of laser induced fluorescence (LIF) was used to measure instantaneous local film thickness on the area of $12 \times 12 \mathrm{~cm}$ with spatial resolution of $0.125 \mathrm{~mm}$.

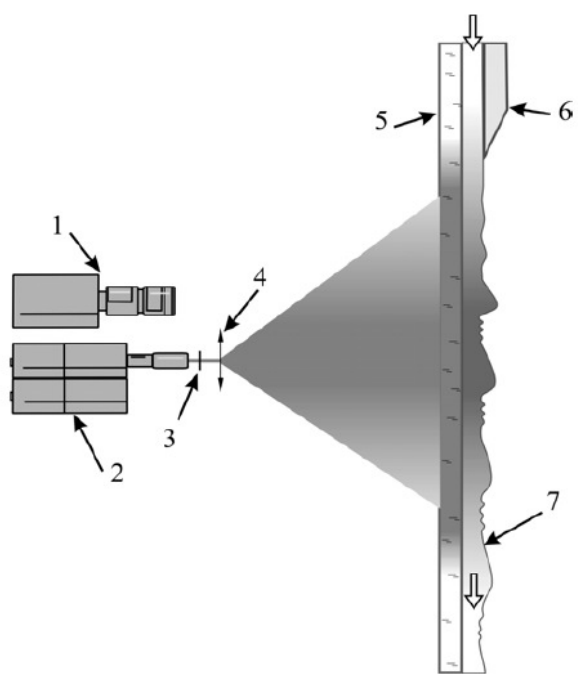

Figure 1. Experimental setup. (1) CCD camera; (2) laser; (3) scattering fluorocarbon film; (4) expanding lens; (5) glass plate; (6) slit distributor; (7) liquid film. White arrows show flow direction.

The LIF method is based on the reconstruction of local film thickness in accordance with local light intensity emitted by a small amount of fluorescent dye dissolved in working liquid and is quite similar to the method of "fluorescent imaging" originally introduced in [3] to study wave dynamics on inclined films. The main difference between the LIF and fluorescent imaging methods is that in the former coherent laser light is used for fluorescence excitation and additional measures should be taken to suppress speckles in illuminated area. As a simple remedy from the speckles, we placed scattering film made of porous fluorocarbon between the laser and expanding lens (Fig. 1). In our experiments working liquids were doped with small additions, about $30-40 \mathrm{mg} / \mathrm{l}$, of fluorescent dye Rhodamine 6 G. To minimize optical distortions arising due to wavy free surface of the film, we arranged the light source and the camera at the dry side of the glass plate, i.e., excitation and registration were carried out through glass. A more detailed description of the method can be found in [4].

\section{Results and discussion}

At moderate Reynolds numbers $R e$, two scenarios of the patterns evolution were observed. For low frequencies of $2 \mathrm{D}$ waves excitation $f$, the leading humps initially run away from the trailing humps. Then their amplitudes and velocities decrease so that the trailing humps which move all the time with approximately constant celerity begin to overtake the leading humps. As the trailing humps approach the leading ones, the latter begin to restore their amplitudes and the pattern structure almost repeats itself through several wave lengths. An example of such oscillatory scenario obtained previously in modeling [5] and observed in experiments [6] is shown in Fig. 2(a). For high excitation frequencies, 
the site between trailing humps, which was left by running downstream leading hump, is filled by another leading hump that arrives from above so that the pattern structure also almost repeats itself through several wave lengths as shown in Fig. 2(b). We will refer to such scenario as "passing through" one.

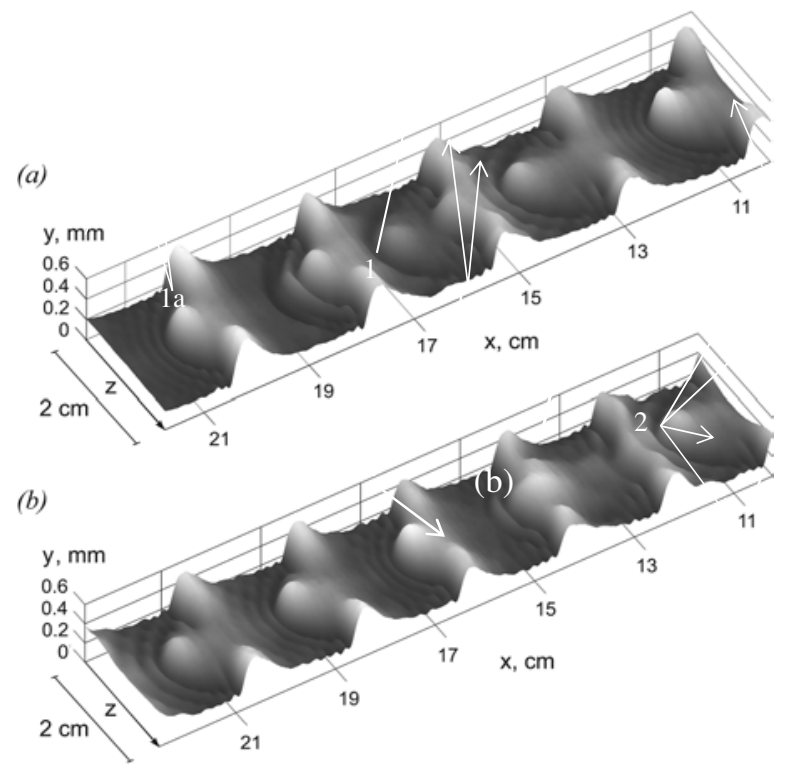

Figure 2. Scenarios of 3D wave patterns evolution. $R e=50$ and $L=2 \mathrm{~cm}$.

(a) $f=17 \mathrm{~Hz}$, oscillatory pattern. (b) $f=20 \mathrm{~Hz}$, passing through pattern.

Figure 3 demonstrates the calculated shape of the developed waves on a WGS rivulet in comparison with experimental data for sufficiently high values of Reynolds number and forcing frequency.
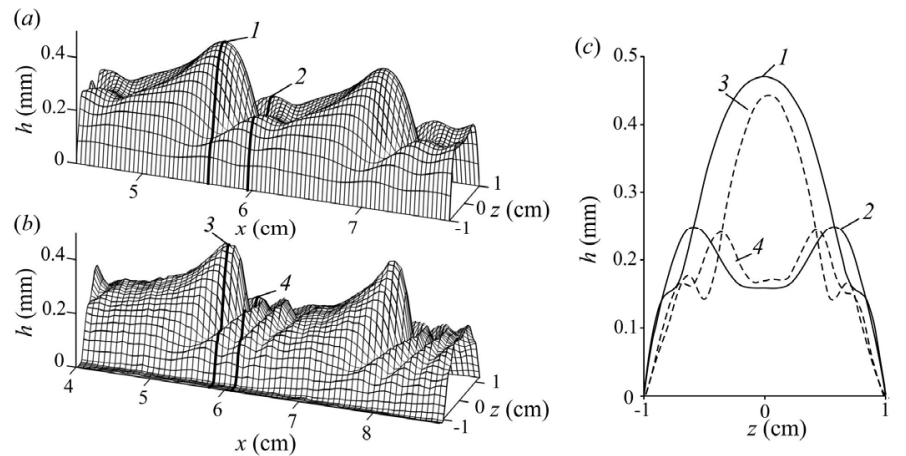

Figure 3. Shape of the wave in the WGS rivulet compared with experimental at $R e=36, f=23 \mathrm{~Hz}$. (a) calculated 3D surface, $(b)$ experimental 3D surface, $(c)$ rivulet cross sections, $(1,2)$ calculation, $(3,4)$ experiment.

As it is clear from the figure, the calculation well describes the shape of the peak with capillary ripples in front of the wave, as well as the rivulet profiles in different cross-sections. Significant discrepancy with the experimental data is observed in the wavelength. The calculated distance between peaks is $1.5 \mathrm{~cm}$, while experiment gives $2 \mathrm{~cm}$. 


\section{Conclusions}

We have investigated experimentally the process of transition from 2D to 3D waves in liquid films falling down a vertical plate with a primary goal to examine to which extent the modern modeling approaches [5, 7] adequately describe real film flows. The method of laser induced fluorescence is used to obtain instant shapes of three dimensional waves and to investigate the regularities of formation of 3D wave patterns arising due to transverse instability of $2 \mathrm{D}$ waves. Although many details of 3D wave patterns correspond well, there are a few significant distinctions between our experiments and modeling. In particular, during 2D-3D wave transition, we observed a strong transverse redistribution of liquid leading to the formation of rivulets on the surface of isothermal liquid film, which is a phenomenon not described previously. Our results show that forming $3 \mathrm{D}$ waves always resemble streak-like waves as in [8] but not $\Lambda$-solitons as in $[9,10]$.

A numerical simulation of nonlinear three-dimensional forced waves in a straight rivulet, flowing down a vertical plate, was carried out. The calculations were performed for the two liquids (WES and WGS) with different physical properties, used in experiments [2]. Compared with the film flow, the waves in rivulets have significant differences. In particular, the wave patterns for the cases of "small" and "large" contact wetting angles are significantly different. The calculations have shown that for WES rivulet ("large" contact wetting angle) the waves are 2D in nature, while in WGS rivulet ("small" contact wetting angle) 3D horseshoe-waves develop. Comparison of experimental data on nonlinear waves with the calculations, made for different values of the Reynolds number within a wide range of the forcing frequency leads to the following conclusions. The applied model [1] is appropriate to describe in general the shape and amplitude of the waves on the rivulet surface at moderate Reynolds numbers. In particular, for WGS rivulet the model predicts waves of step-wise shape, which were observed in the experiments at low forcing frequencies. For moderate forcing frequencies the model predicts waves with capillary ripples in front of the wave peak. The shape of these waves is in good agreement with the experimental data. For WES rivulet the model predicts well the amplitude of developed waves and the shape of the rivulet wave surface. Besides, for WES rivulet the model predicts no capillary ripple in front of the main peak that is consistent with the experimental data.

\section{Acknowledgements}

The work was supported by Russian Scientific Foundation (projects 15-01-06702 and 15-01-04320).

\section{References}

1. E.A. Demekhin, V.Y. Shkadov, Fluid Dynamics. 19, 689 (1984).

2. S.V. Alekseenko, A.V. Bobylev, V.V. Guzanov, D.M. Markovich, S.M. Kharlamov, Thermophys. Aeromech., 17, 371 (2010).

3. J. Liu, J. D. Paul, and J. P. Gollub, J. Fluid Mech. 250, 69 (1993).

4. S.M. Kharlamov, V.V. Guzanov, A.V. Bobylev, S.V. Alekseenko, D.M. Markovich, Phys. Fluids, 27, 114106 (2015).

5. G.F. Dietze, W. Rohlfs, K. Nährich, R. Kneer, B. Scheid, J. Fluid Mech. 743, 75 (2014).

6. S.V. Alekseenko, V.V. Guzanov, D.M. Markovich, S.M. Kharlamov, Tech. Phys. Lett. 38, 739 (2012)

7. B. Scheid, C. Ruyer-Quill, P. Manneville, J. Fluid Mech. 562, 183 (2006).

8. P. Adomeit and U. Renz, Int. J. Multiphase Flow, 26, 1183 (2000).

9. E.A. Demekhin, E.N. Kalaidin, S.Kalliadasis, S.Yu. Vlaskin, Phys. Rev. E, 82, 036322 (2010).

10. E.A. Demekhin, E.N. Kalaidin, A.S. Selin, Phys. Fluids, 22, 092103 (2010). 